\title{
Musikbibliothek im europäischen Kontext
}

\section{Zur Entstehung der Musiksammlung der Österreichischen Nationalbibliothek}

Die großen historisch-wissenschaftlichen Bibliotheken Europas weisen eine ähnliche organisatorische Struktur auf, und eines dieser Strukturmerkmale ist die Untergliederung in thematisch bestimmte Spezialabteilungen bzw. -sammlungen, ob es sich nun um Handschriften, Karten, Bilder, Papyri oder Musikalien handelt. Die Gründung - oder meist eher: Entstehung - der meisten Musikabteilungen liegt im 19. Jahrhundert und weist erstaunliche Parallelen auf, sowohl was den Zeitpunkt als auch was die historischen Voraussetzungen und die Bestandsschwerpunkte betrifft. Dies sei in Kürze am Entstehungsprozess von Musikabteilungen an vier bedeutenden Bibliotheken des deutschen Sprachraums gezeigt, der Staatsbibliothek zu Berlin, der Sächsischen Landesbibliothek - Staats- und Universitätsbibliothek Dresden, der Bayerischen Staatsbibliothek in München und der Österreichischen Nationalbibliothek in Wien.

\section{Entstehung von Musiksammlungen}

Berlin. Die im Internet bezüglich der Entstehung der Musikabteilung veröffentlichte Selbstbeschreibung lautet:

„Musikabteilungen oder Musiksammlungen an Bibliotheken sind eine recht junge Erscheinung, die erst im 19. Jahrhundert greifbar werden. Zu einer der am frühesten begonnenen Sammlungen gehört jedoch diejenige in Berlin. Erwerbungen wie die geschlossene Musikaliensammlung des Hallenser Universitätsmusikdirektors Johann Friedrich Naue, die 1824/25 in die Königliche Bibliothek kam, oder die umfangreiche Musikbibliothek von Georg Pölchau mit 1.879 Drucken und 2.647 Abschriften und Autographen - in der sich auch zahlreiche Autographen Johann Sebastian Bachs befanden und die im Jahr 1841 angekauft wurde - führten dazu, dass im Mai 1842 mit Siegfried Dehn ein eigener Kustos für das Fach Musik eingestellt wurde. Damit waren die Voraussetzungen für die planmäßige Erwerbung von Musikalien und Fachliteratur im Rahmen einer eigenen musikalischen Abteilung geschaffen. Unter Dehn erwarb die Bibliothek u.a. 1846 viele Beethoven-Au- 
tographen aus dem Nachlass Anton Schindlers und 1854 weitere Bach-Autographen und andere Abschriften aus dem Umfeld Bachs aus dem Archiv der Sing-Akademie zu Berlin. In den 60er Jahren des 19. Jahrhunderts übergab die Intendanz der Königlichen Schauspiele Berlin etwa 1300 Opernpartituren und Aufführungsmaterialien, die bis heute in der Musikabteilung der SBB verwahrt werden."

Dresden. Zu ihrer Musikabteilung bzw. deren Entstehung merkt die Sächsische Landesbibliothek Staats- und Universitätsbibliothek Dresden an:

„1816: Oberbibliothekar Friedrich Adolf Ebert vereinigt die im Bestand verstreuten Musica theoretica und practica der damaligen Königlichen Öffentlichen Bibliothek zu einer, Musicalischen Abtheilung. 1890: Die Bibliothek erhält Musikalien des 16. und 17. Jahrhunderts aus sächsischen Kirchen-, Schul- und Ratsbibliotheken als Depositum. 1896: König Albert weist der Bibliothek die Königliche Privat-Musikaliensammlung zu. 1908 folgen die Hofkapell-Musikalien aus der Katholischen Hofkirche. 1934: Die Musikabteilung der nunmehrigen Sächsischen Landesbibliothek übernimmt erstmals Musikalien aus dem historischen Fundus der Staatsoper." ${ }^{\text {"2 }}$

München. Hier wird darauf hingewiesen, dass es bereits vor der (relativ späten) Etablierung einer Musikabteilung eine rege Erwerbungstätigkeit von Musikalien gab:

„Die historischen Grundbestände der Musikabteilung gehen zurück auf die Musikalien der Hofbibliothek und der Bayerischen Hofkapelle, die im 16. Jahrhundert europäischen Rang besaß. Es ist sehr ungewöhnlich, dass die bayerischen Herzöge schon damals gezielt musikalische Quellen für ihre Hofbibliothek sammelten, unabhängig vom Aufführungsmaterial, das die Hofkapelle benötigte. Auf diese Weise wurden die Grundlagen dafür geschaffen, dass die Bayerische Staatsbibliothek heute überaus reiche Bestände aus dem 16. Jahrhundert besitzt (z.B. mehr als 1.400 Notendrucke). Im 17. und 18. Jahrhundert wuchsen die Musikalien der Hofkapelle jedoch wesentlich stärker als diejenigen der Hofbibliothek. Kern dieser Kapellbestände sind die insgesamt 75 erhaltenen Chorbuch-Manuskripte im Folio-Format: eine im In- und Ausland einmalige Sammlung, die 1523 unter Ludwig Senfl begonnen wurde und unter Orlando di Lasso ihren Höhepunkt erreichte. Hiervon zu unterscheiden sind die kostbaren Handschriften, die zum persönlichen Besitz der bayerischen Herzöge und Kurfürsten gehörten. Aufgrund der Bestandserweiterung durch Erwerbung und Eingliederung von umfangreichen Musiksammlungen seit der Mitte des 18. Jahrhunderts wurde im 1843 fertiggestellten Bibliotheksgebäude an der Ludwigstraße 1857 eine eigene ,Musikalische Abteilung' gegründet, deren Konservator Julius Joseph Maier die handschriftlichen und gedruckten Bestände in Katalogen vertieft erschloss, die bis weit in das 20. Jahrhundert hinein mustergültig waren. Maier initiierte

1 http://staatsbibliothek-berlin.de/die-staatsbibliothek/abteilungen/musik/geschichte (3.9.2015).

2 http://www.slub-dresden.de/sammlungen/musik/ueber-die-musikabteilung/geschichte-der-musikabteilung (20.1.2016). 
auch die kontinuierliche Erwerbung und Ergänzung historischer und zeitgenössischer Musikhandschriften und der neu erscheinenden Notendrucke und Bücher über Musik.."

Wien. Die Entstehung einer Musikabteilung in der Wiener kaiserlichen Hofbibliothek ist eng mit dem Wirken des Grafen Moritz von Dietrichstein verbunden. Dietrichstein wurde nach kurzer militärischer Karriere 1819 zum Hofmusikgrafen ernannt; als solcher hatte er die Aufgabe, die praktisch-musikalischen Belange des Wiener Kaiserhofs zu betreuen, somit die Hofkapelle, die Hofkirchenmusik und die Hofmusik zu organisieren. In dieser Funktion machte er die Erfahrung, dass sich das Hofmusikarchiv, das ausschließlich als Sammlung von Aufführungsmaterial verstanden wurde, in verwahrlostem Zustand befand und der besonderen Zuwendung bedurfte. Als Dietrichstein 1826 mit der Leitung der Hofbibliothek betraut wurde, sorgte er sogleich für die Überführung der Bestände des Hofmusikarchivs in die Hofbibliothek, für die Vereinigung mit den bereits in der Hofbibliothek befindlichen Musikalien und für deren Katalogisierung, die 1831 abgeschlossen wurde. Damit war der Grundstock für die Musiksammlung innerhalb der Hofbibliothek (der heutigen Österreichischen Nationalbibliothek) gelegt, wenngleich diese noch keine organisatorische Eigenstruktur besaß.

\section{Voraussetzungen: Kunstwerkscharakter und neue Rangstufe der Musik}

Ein vergleichender Blick auf die Entstehung von Musiksammlungen an den genannten Bibliotheken zeigt eine erstaunliche chronologische und inhaltliche Koinzidenz: Durchwegs liegt diese Entstehung in der ersten Hälfte des 19. Jahrhunderts. Bemerkenswert ist weiters, dass in diesem Zeitraum Aufführungsmaterialien der jeweiligen höfischen Trägerinstitution in die Bibliothek integriert wurden, da man sie nunmehr als wichtige historische Quellen erkannte. Dies als Zufall abzutun, würde zu kurz greifen: Es lässt sich anhand mehrerer historischer Aspekte und deren Interaktion zeigen, dass wir es mit der folgerichtigen Konsequenz einer geistesgeschichtlichen Entwicklung zu tun haben.

\section{Der Kunstwerkscharakter von Musik}

Der Begriff des klassischen musikalischen Kunstwerks (klassisch nicht im stilgeschichtlichen Sinn, sondern im Sinn eines als überzeitlich angesehenen Geltungsanspruchs) ist dem Angehörigen der europäischen Musikkultur des 19., 20. und 21. Jahrhunderts so vertraut geworden, dass ihm kaum noch zu Bewusstsein kommt, dass dieses musikalische Kunstwerk im emphatischen Sinn weder in seiner eigenen Kultur immer schon als Gegebenheit galt, noch dass es anderen Kulturen in dieser Weise unbekannt ist. Die Vorstellung, dass Musik nicht nur für einen bestimmten Anlass, nicht nur für ein hic et nunc geschrieben und ausgeübt werden solle, dass sie also mehr sein könne als ,Gebrauchs-' oder ,Umgangsmusik‘ im Dienste sakraler oder weltlich-repräsentativer Ereignisse diese Vorstellung hat sich im Laufe der Geschichte erst langsam herausgebildet. So problematisch

3 https://www.bsb-muenchen.de/die-bayerische-staatsbibliothek/abteilungen/musikabteilung/bestaende/ (8.1.2016). 
es ist, historische Umschichtungsprozesse punktuell zu datieren, wird man doch die Schwelle dieses Bewusstseinswandels etwa in der zweiten Hälfte des 18. Jahrhunderts ansetzen dürfen, in einem Zeitraum, der nicht nur auf dem Gebiet der Musik zu einer geistigen Neuorientierung fundamentaler Art führte. Es ist dies zugleich der Zeitraum, in dem die Betrachtungsweise wirksam zu werden beginnt, die wir als „historisch“ bezeichnen. Sie besagt, dass Vergangenes bewusst in seinem Anderssein erkannt wird, zugleich aber auch in seiner geschichtlichen Determiniertheit.

Die neue Bedeutung des musikalischen Textes

Mit dem wachsenden Geschichtsbewusstsein kommt der Gedanke auf, auch musikalische Werke - gleich dichterischen und bildnerischen - als individuelle Genietaten anzusehen, die ihren Schöpfer überleben, und nicht bloß als Gattungsexemplare eines bestimmten (meist funktionsgebundenen) Stils. War bisher das Aufführungsereignis die zentrale Kategorie, welcher der Text als Vorlage diente, so kehrt sich bei Beethoven das Verhältnis um; seine Werke stellen - durchaus auch im Bewusstsein ihres Schöpfers - unantastbare musikalische ,Texte dar, deren Sinn durch Interpretationen, die als ,Auslegungen` zu verstehen sind, entschlüsselt werden soll.

„Daß ein Komponist, der sich um die ,elende Geige des Ignaz Schuppanzigh, wie Beethoven sie nannte, nicht kümmerte, den Anspruch durchsetzen konnte, daß die Aufführung eine Funktion des Textes und nicht der Text eine Funktion der Aufführung sei, sollte, obwohl es unter ästhetisch Gebildeten zur Selbstverständlichkeit geworden ist, von Historikern als das frappierende Ereignis nachempfunden werden, das es für die Zeitgenossen im frühen 19. Jahrhundert gewesen sein muß. Daß in einem musikalischen Text - ähnlich wie in einem dichterischen oder einem philosophischen - eine Bedeutung aufbewahrt ist, die sich zwar durch eine tönende Darstellung sinnfällig machen läßt, in ihr jedoch nicht aufgeht, daß also ein musikalisches Gebilde ein ,Ideenkunstwerk' sein kann, das als Text jenseits der Auslegungen ideellen Bestand hat, war das eigentlich Neue, das durch Beethoven dem ästhetischen Bewußtsein des Zeitalters aufgezwungen worden ist." ${ }^{\text {"4 }}$

Hat nun ein Text diese gehobene ideelle Bedeutung, so kommt auch seiner schriftlichen Fixierung und deren Aufbewahrung ein neuer Stellenwert zu.

Die „physiognomische“ Dimension der Handschrift

Ebenfalls um 1800 wurde die Handschrift - besser gesagt, der Charakter der Handschrift - erstmals als Erkenntnisquelle für die Individualität des Künstlers interpretiert. Anregungen dazu gab Johann Caspar Lavaters großangelegtes Werk Physiognomische Fragmente, ${ }^{5}$ dessen französischer Ausgabe von 1806 der Herausgeber Moreau de la Sarthe ein neues Kapitel anschloss: Reflexions sur les caractères physiognomiques tirés de l'écriture. 1812 erschien das erste selbstständige Buch

4 Carl Dahlhaus, Die Musik des 19. Jahrhunderts, Wiesbaden 1980, S. 9 (Neues Handbuch der Musikwissenschaft. 6).

5 Johann Caspar Lavater, Physiognomische Fragmente, Leipzig u. a. 1775-1778, Bd. 1-4. 
über Graphologie (Edouard Hocquart, L'Art de juger de l'esprit et du charactère des hommes sur leur écriture). $\mathrm{Zu}$ dieser Zeit war das Sammeln von Originalhandschriften in literarischen Kreisen bereits durchaus verbreitet. Als einer der ersten und zugleich prominentesten Autographensammler begegnet uns Johann Wolfgang von Goethe; angeregt durch Christoph Gottlieb Murrs Werk Chirographia personarum celebrium (1804) begann er „chirographa von bedeutenden Männern alter und neuer Zeit“ zu erwerben. An Johann Friedrich Blumenbach schrieb er 1805:

„Haben Sie doch ja die Gefälligkeit, von Zeit zu Zeit an meine fromme Sammlung zu denken: denn fromm ist doch wohl Alles, was das Andenken würdiger Menschen zu erhalten und zu erneuern strebt [...]. Vielleicht lasse ich ein compendiöses Register meiner Sammlung drucken, um meine auswärtigen Freunde zu gefälligen Beiträgen anzuregen. ${ }^{\text {“6 }}$

Dass in diesem „Andenken würdiger Menschen“ durchaus auch Komponisten eingeschlossen waren, zeigt ein Brief Goethes an Karoline Pichler (1812):

„Wenn von der eigenen Hand des vortrefflichen Mozart sich Ihren Bemühungen keine Zeit darbot, [...] so werde ich um desto eifriger sammeln, weil uns dieses Beispiel zeigt, wie gerade das Nächste und Eigentümlichste des Menschen so bald nach seinem Scheiden verschwindet und von seinem Zustande, wie von seinen Verdiensten, nur ein Allgemeines, gleichsam Körperloses übrig bleibt. “7

In diesem Falle war Goethe zu spät gekommen; bereits 1799 hatte der Offenbacher Verleger Johann André den handschriftlichen Nachlass Mozarts von dessen Witwe Constanze erworben.

Die neue gesellschaftliche und ideelle Stellung des Komponisten

Der frappierende soziale Aufstieg des Komponisten um die Wende vom 18. zum 19. Jahrhundert vom Hofbediensteten (Haydn) zur autonomen künstlerischen Instanz (Beethoven) - ist ein unmittelbares Korrelat zur Stellung, die nunmehr die Musik im Kanon der Künste einnahm. Nachdem Musik jahrhundertelang funktional gebunden und somit ,dienend ' gewesen war, verwirklichte sich nun in der reinen Instrumentalmusik die „Idee der absoluten Musik“ als einer von vordergründigen Funktionen und Zweckbestimmungen ,losgelösten', geistigen Aussage, die nicht nur neben, sondern sogar über das bisher dominierende literarische Kunstwerk gestellt wurde. (Man denke etwa an die Beethoven-Rezensionen E. T. A. Hoffmanns oder an die exzeptionelle Stellung, die Arthur Schopenhauer in seiner Welt als Wille und Vorstellung der Musik im Kanon der Künste einräumte.) Musik war nun nicht mehr bloß gediegenes Kunsthandwerk, bewertbar anhand der aufgestellten Regeln, sondern Vermittlung einer „Ahnung des Unendlichen“, formuliert in einer Sprache, die das ausdrückte, was „Worte nicht mehr vermögen“.

6 Albi Rosenthal, „Tradition des Autographensammelns, historisch“, in: Internationales Symposium Musikautographe. 5.-8. Juni 1989, Wien; Bericht, redigiert von Ernst Hilmar, Tutzing 1990, S. 19 (Publikationen des Instituts für Österreichische Musikdokumentation. 16).

7 Ebd. S. 19 f. 
All dies - der allgemein akzeptierte Kunstwerkscharakter von Musik und die damit verbundene Wandlung musikalischer Texte von Aufführungsmaterialien zu historischen Dokumenten, der physiognomische Charakter von Handschriften, die neue gesellschaftliche Stellung der Komponisten und das entwickelte bürgerliche Konzertleben - führte dazu, dass (mit leichter Verspätung) in den Bibliotheken musikalische Texte den literarischen und wissenschaftlichen Texten im Rang gleichgestellt wurden, was die Herausbildung musikalischer Abteilungen zur Folge hatte. Freilich sind solche Prozesse nie abstrakt, sondern mit dem Wirken konkreter Persönlichkeiten verbunden. In Wien stehen hier Gottfried van Swieten und Moritz Graf von Dietrichstein im Blickpunkt, deren Lebenswege in vielfacher Weise mit dem Musikleben Wiens verflochten sind. Zugleich sei dargestellt, in welcher Weise sich bereits ab dem späten 18. Jahrhundert das erwachende historische Interesse auf dem Gebiet der Musik im Bereich der Wiener Hofbibliothek manifestierte. ${ }^{8}$

\section{Das Beispiel Wiener Hofbibliothek - Musiksammlung der Österreichischen Nationalbibliothek}

Musikalischer Historismus im Umfeld der Wiener Hofbibliothek

Erste Ansätze zu einer gezielten Pflege musikalischer Belange unter historischem Aspekt sind im Wirkungsbereich Gottfried van Swietens festzustellen, der von 1777 bis 1803 Präfekt der Hofbibliothek war. Van Swieten hatte als Diplomat in England die Musik Georg Friedrich Händels, insbesondere dessen große Oratorien, kennengelernt und Partituren der Werke erworben. Er selbst hatte musikalische Ambitionen und komponierte, wiewohl sich Haydn über seine diesbezüglichen Versuche nicht sehr schmeichelhaft äußerte. Auf das Musikleben Wiens hatte er einen nicht unbedeutenden Einfluss; seine 1785 gegründete „Gesellschaft der associierten Cavaliers“ veranstaltete zahlreiche Aufführungen von Oratorien. Auch in seiner Dienstwohnung in der Hofbibliothek sowie im Prunksaal fanden auf Initiative Van Swietens Aufführungen, hauptsächlich von Werken Händels, statt, die Joseph Starzer und nach 1787 Wolfgang Amadeus Mozart für diesen Zweck bearbeiteten und leiteten.

Als Besitzer einer eigenen Musikbibliothek und als Komponist schenkte Van Swieten der Musik auch in seiner Tätigkeit als Bibliothekspräfekt besondere Beachtung. Zweifellos unter ,musikalischem Aspekt' nahm er 1792 Carl Leopold Röllig als Bibliotheksdiener auf. Röllig, ein gebürtiger Hamburger (geboren um 1755), hatte ein unruhiges künstlerisches Wanderleben als Glasharmonikavirtuose, Komponist und Erfinder neuer Musikinstrumente (Orphika, Xenorphika) hinter sich; er kam 1791 nach Wien und war offensichtlich froh, sich nun ruhigen und stetigen Tätigkeiten widmen zu können. Van Swieten gab ihm den Auftrag, die verstreuten musikalischen Bestände der Bibliothek zusammenzufassen und in eine systematische Ordnung zu bringen; gezielte Erwerbungstätigkeit auf musikalischem Gebiet hatte bis dahin nicht stattgefunden. Im Zeitraum von zehn Jahren führte Röllig diese Aufgabe gemäß den Intentionen des Präfekten durch. Dieser starb 1803 ohne Hinterlassung eines Testamentes; in seinem Fall hätte die Hofbibliothek erstmals die Chance der Erwerbung eines hochkarätigen musikalischen Nachlasses gehabt, denn Van Swieten

8 Franz Grasberger, Die Musiksammlung der Österreichischen Nationalbibliothek, Wien 1978. 
besaß als Textdichter der Schöpfung und der Jahreszeiten die Originalpartituren Joseph Haydns. Über das Schicksal der 1804 versteigerten Bibliothek ist bedauerlicherweise wenig bekannt; nicht einmal ein Exemplar des von Röllig erstellten Auktionskataloges blieb erhalten.

Gottfried van Swieten war jedoch nicht der einzige, der sich im Wien des späten 18. Jahrhunderts musikalischer Sammeltätigkeit widmete. In diesem Zeitraum entstanden auch andere musikbezogene Sammlungen, etwa die des Baron Joseph Philipp du Beyne de Malechamp, des Franz Bernhard Ritter von Keeß oder des Franz Joseph Ritter von Heß. Sie sind die Vorläufer der Privatmusiksammlungen des Vormärz, die mit bekannten Persönlichkeiten des Wiener Kulturlebens verbunden sind: die Namen Joseph Sonnleithner, Simon Molitor, Kaiser Franz I. (II.), Erzherzog Rudolph, Raphael Georg Kiesewetter und Aloys Fuchs stehen für diese historistischen Initiativen. Angeregt durch die Sammeltätigkeit kam es zu zahlreichen Aufführungen von Vokalund Instrumentalwerken alter Meister des 16. bis 18. Jahrhunderts; die Wiener Pflege der Werke Bachs und Händels hatte hier ihre Basis. Es war somit nicht nur die Präsenz Ludwig van Beethovens, die 1812 Louis Spohr veranlasste, Wien als die unbestrittene Hauptstadt der musikalischen Welt zu bezeichnen.

1812 ist das Gründungsjahr der Gesellschaft der Musikfreunde in Wien; diese nahm bereits in ihre ersten Statuten von 1814 wissenschaftliche und museale Aspekte auf und legte damit den Grundstein zu der herausragenden Sammlung, die das Archiv der Gesellschaft bis heute darstellt. Auf historistischen Bestrebungen beruhte auch der Plan des Gründers Joseph Sonnleithner, alte Musik neu zu edieren. Bereits im Jahre 1800 wurde der Plan zu einer Geschichte der Musik in Denkmälern von der ältesten bis auf die neueste Zeit gefasst, den Johann Georg Albrechtsberger, Joseph Haydn und Antonio Salieri aktiv unterstützten. 1803 war in Wien ein Band bereits gestochen, dann machten die Kriegswirren dem Unternehmen ein vorläufiges Ende: Die 1805 in Wien eingerückten Franzosen schmolzen die Platten ein, um daraus Munition herzustellen. Aber der ,Denkmälergedanke‘ war geboren, blieb lebendig und führte im späteren Verlauf des Jahrhunderts zu den bekannten Denkmäler- und Gesamtausgaben.

\section{Moritz Graf von Dietrichstein (1775-1864)}

Im Kontext dieser Umgebung, die der musikalischen Vergangenheit bereits vielfache Beachtung schenkte, ist die Tätigkeit Moritz Graf von Dietrichsteins zu sehen. Nach einer kurzen militärischen Laufbahn widmete sich Dietrichstein vornehmlich pädagogischen und künstlerischen Tätigkeiten; in seinem Hause verkehrten Dichter, Musiker, Maler und Bildhauer. Seit der Gründung der Gesellschaft der Musikfreunde war Dietrichstein Mitglied dieser Vereinigung und wurde $1815 \mathrm{zu}$ ihrem ersten Vizepräses gewählt. Vom Wiener Hof erhielt er die Aufgabe, die Erziehung des Herzogs von Reichstadt zu übernehmen, des Sohnes aus der Ehe Napoleons mit der österreichischen Kaisertochter Marie Louise; diese Verpflichtung währte von 1815 bis 1831.

Am 26. August 1819 wurde Moritz Graf von Dietrichstein zum „Hofmusikgrafen“ ernannt. Dies war eine vornehmlich administrative Funktion bei Hof; Dietrichstein hatte nun die Aufgabe, die Hofkapelle, die Hofkirchenmusik und die Hofmusik zu betreuen, den Einsatz der Sängerknaben und Musiker zu leiten und das Hofmusikarchiv zu verwalten. Diese Sammlung 
erweckte seine besondere Aufmerksamkeit, handelte es sich doch um die vielfältigen Zeugnisse der Musikausübung am Wiener Kaiserhof, zurückreichend bis in das 17. Jahrhundert. Über die Auffindung und über den Zustand dieses Bestandes berichtete Dietrichstein 1829 an das Wiener Obersthofmeisteramt:

"Als mir im Monate September 1819 das Hofmusikgrafenamt anvertraut wurde, war meine erste Sorge, die mangelnde Ordnung in allen Zweigen dieses Hofdienstes herzustellen und vorzügliche Rücksicht dem Hofmusik-Archive zu schenken. Ich traf dasselbe in einem Zustande völliger Verwahrlosung. Es befand sich, wie bekannt, in einigen Kästen zwischen dem physikalischen Kabinette des (damaligen) Herrn Abbé Stelzhammer und den Oratorien der Hofkapelle. Ein großer Theil der Hauptschätze dieses Archives war in einem Mauerloche (!) zwischen zwei Oratorien verborgen, in dem es seit mehr als dreißig Jahren lag, ohne dass man daran dachte, diese Überreste einer, unter S. M. dem Kaiser Joseph II. [...] viel größeren und in jener Zeit, (wie mir der verstorbene Hofkapellmeister Salieri erzählte) auf höheren Befehl sortierten und theilweise an den Kunstfeuerwerker im Prater abgelieferten Sammlung, ans Tageslicht zu fördern, besonders zu ordnen und ihr einen angemessenen Platz anzuweisen. Die Kataloge sowohl der alten, als neuen Kirchenund sonstigen Musik waren sehr mangelhaft, undeutlich und zeigten, wie gering man die musikalischen Schätze beachtete, die von mehreren, der Tonkunst besonders ergebenen deutschen Kaisern hinterlassen wurden und wie viel durch die Gleichgültigkeit und Nachlässigkeit ihrer Aufseher, wie durch die Gewohnheit: Das anscheinend Unnütze zu vernichten, verschwunden und nunmehr unwiederbringlich verloren seyn mag."

Die inhaltlichen Schwerpunkte dieser Sammlung, über die noch gesondert zu sprechen sein wird, waren durch das Musikinteresse der komponierenden Habsburgerkaiser Ferdinand III., Leopold I., Joseph I. und Karl VI. bestimmt.

Mit einer Hingabe, die wohl durchaus über die unmittelbare dienstliche Verpflichtung hinausging, widmete sich nun Dietrichstein dem Hofmusikarchiv und plante, es zu einer öffentlich zugänglichen Sammlung zu machen. In dieser Intention ließ er sich von zeitgenössischen Fachleuten beraten: von Raphael Georg Kiesewetter, der Pionierarbeit in der Erforschung der Periode der Niederländer leistete und seine eigene Sammlung nach musikgeschichtlichen Prinzipien aufbaute, vom Skriptor der Hofbibliothek Anton Schmid, der hier ab 1818 als musikalischer Sachbearbeiter tätig war, und auch vom Komponisten Ignaz Franz von Mosel, mit dem Dietrichstein eine langjährige Freundschaft verband. Es war Dietrichsteins Absicht, „die reichen und seltenen musikalischen Schätze für talentvolle angehende Tonsetzer nützlich und für Gelehrte im musikalischen Fach zugänglich zu machen. ${ }^{\text {10 }}$ Diese Pläne traten jedoch etwas in den Hintergrund, als Dietrichstein ab 1821 neben dem Hofmusikgrafenamt auch die Direktion der Hoftheater übernehmen musste.

9 Wilhelm Nemecek, Moritz I., Graf von Dietrichstein (1775-1864). Hofbibliothekspräfekt (1826-1845), Phil. Diss. Wien 1953, S. 99.

10 Franz Grasberger, Die Musiksammlung 1978 (wie Anm. 8), S. 8. 
„Meine Theatergeschäfte und alle jene, die mir zu besorgen oblagen, hinderten mich jedoch, diesen Anstalten die nöthige wissenschaftliche Vollendung zu geben, mit der sich die Absicht verbunden hatte, diese reichen, seltenen Vorräthe für eine dereinstige Geschichte der Musik in Österreich brauchbar [...] zu machen. “"11

Es war daher bald klar, dass für das Hofmusikarchiv eine stabile Bleibe gefunden werden musste, und dies bedeutete, „den ganzen, dem eigentlichen Kirchendienst fremden Theil des HofmusikArchivs einem anderen, sicheren, keinen Veränderungen unterworfenen, kaiserlichen literarischen Institute zuzuwenden. ${ }^{12}$ Damit konnte keine andere Institution als die kaiserliche Hofbibliothek gemeint sein.

Der weitere Verlauf der Ereignisse kam Dietrichsteins Intentionen entgegen. Mit Entschließung vom 30. Mai 1826 enthob ihn Kaiser Franz I. seiner bisherigen Ämter und ernannte ihn zum Präfekten der Hofbibliothek, die zu dieser Zeit zu den bedeutendsten Bibliotheken der Welt gezählt werden durfte. In seiner neuen Funktion konnte Dietrichstein nun die bereits gefassten Entschlüsse umsetzen: Bereits am 11. Juni, knapp zwei Wochen nach Amtsantritt, beantragte er die Überlassung der älteren Bestände des Hofmusikarchivs, was drei Tage darauf genehmigt wurde. Anton Schmid übernahm diesen ersten Teil des Hofmusikarchivs, der die wertvollen Musiksammlungen der komponierenden Barockkaiser enthielt.

In dem Bericht, den Dietrichstein 1829 für das Obersthofmeisteramt verfasste und in dem er zusammenfassend seine Bemühungen um die Schaffung einer kohärenten Musikaliensammlung darstellte, ist von dem Problem die Rede, dieser neuen Sammlung innerhalb des zur Verfügung stehenden Raumes - es handelte sich nach wie vor bloß um den großen barocken Bibliothekssaal aus der Zeit Karls VI. - eine sinnvolle Aufstellung zu ermöglichen:

„Die Anschaffung der 64 Kästen erleichterte das Verwirklichen des schon früher genährten Wunsches: [...] die in dem Büchersaale [...] zerstreuten, theoretischen und praktischen musikalischen Werke auf einem Punkte zu vereinigen, - eine Verfügung, welche der Übersicht und Benützung derselben gleich vortheilhaft ist.“

Anton Schmid wurde beauftragt, diese Vereinigung durchzuführen, und Dietrichstein konnte 1829 zufrieden vermelden:

„Diese bedeutende Sammlung alter, höchst seltener, zum Theil an keinem anderen Orte zu findenden, theoretischen musikalischen Werke gewährt mir die angenehme Erfahrung, dass sie zu den reichsten in Europa gezählt zu werden verdiene, worauf die k.k. Hofbibliothek stolz ist. ${ }^{\text {13 }}$

In der Literatur zur Entstehung der Musiksammlung der Österreichischen Nationalbibliothek wird dieser Satz meist als „Gründungsurkunde“ der Sammlung apostrophiert, was aber auf einem

11 Nemecek, Moritz I. 1953 (wie Anm. 9), S. 100.

12 Ebd.

13 Ebd. 
Missverständnis beruht, denn Dietrichstein spricht hier zunächst nur von der räumlichen Konzentration der bereits in der Hofbibliothek vorhandenen musiktheoretischen Werke. Die Entstehung einer genuinen Musiksammlung erfolgte erst in den Folgejahren durch die Erschließung der Bestände des Hofmusikarchivs, dessen Transfer in die Hofbibliothek Dietrichstein als einen Akt der Bewahrung, vor allem aber der Ermöglichung von Benützung durch Musiker und Gelehrte beschreibt:

„Das Hofmusikarchiv wird niemals, - die Hofbibliothek aber von allen Gattungen Wissbegieriger besucht und so werden in ihr alle Quellen erforscht, aus denen wissenschaftliches und künstlerisches Streben Rath und Befriedigung zu schöpfen hofft. Tonsetzern und musikalischen Gelehrten konnte bisher keine genügende Antwort auf ihre Anfragen gegeben werden, weil die theoretische Sammlung zerstreut war und für mangelhaft galt, eine ihr entsprechende praktische aber an klassischen Werken noch zu unbedeutend ist. ${ }^{\text {"14 }}$

Auf Dietrichsteins Veranlassung wurden unter den „neuen Kästen“ (Schränke), die der Aufnahme von Neuerwerbungen dienten, 16 für Musikalien reserviert. Gemäß der zeitgenössischen Gepflogenheit, streng lokal gebundene Aufstellungssignaturen (sogenannte „Kastensignaturen“) zu vergeben, erhielten die Musikalien des Hofmusikarchivs Signaturen mit dem Kürzel „AN“ (= Armaria nova). Bereits 1831 konnte Dietrichstein berichten, dass die musikalische Sammlung „in 16 der neuen Kästen vereinigt und systematisch geordnet ${ }^{\text {"15 }}$ worden sei. Besonderes Lob wurde dem musikkundigen Scriptor Anton Schmid gezollt:

„Dem Eifer des Scriptors Schmid gelang es in dem verhältnismäßig kurzen Zeitraum, neben seinen sonstigen currenten Arbeiten - nicht nur diese Zusammenstellung, sondern auch schon ein vollkommenes Standorts-Repertorium aller 16 Kästen und einen Zettelkatalog über 15 derselben zu Stande zu bringen, welches mehr als 8000 Zetteln, worauf die Werke genau beschrieben sind, in sich begreift. "16

Schmid freilich musste bei der Revision der bereits in der Hofbibliothek vorhandenen, meist in Stimmensätzen überlieferten alten Musikdrucke feststellen, dass man bei der Übernahme und Bewahrung nicht sehr sorgfältig umgegangen war und zahlreiche Stimmen fehlten. In seiner Meldung an Dietrichstein vom 21. September 1832 konnte er sich einen Seitenhieb auf das mangelnde musikhistorische Bewusstsein seiner Vorgänger, insbesondere zur Zeit der josephinischen Klosteraufhebungen, nicht versagen:

„[...] unter unserer älteren Musik gibt es wohl noch über 200 Werke, zu denen bald die eine, bald die andere, oder gar mehrere Stimmen fehlen. Ich möchte darüber meinen, dass man auf diese Reliquien der Kunst aus den verflossenen Jahrhunderten in früherer Zeit so wenig achtete, die bereits vorhandenen vollständig zu erhalten, theils zur Vermehrung

14 Ebd., S. 101. Unterstreichungen sind original.

15 Ebd.

16 Ebd. 
gar nichts beitrug, wo doch wohl durch die Aufhebung der Klöster in Österreich gewiss Gelegenheit genug vorhanden war. [...] Da mag wohl, wie man mir gar oft erzählt hat, durch die stupende Ignoranz und Indolenz der damit beauftragten Kommissäre mancher schöne Codex und manches interessante Druckwerk entweder zum Kässtecher, oder in die Hände gieriger Privat-Sammler, nach dem Preis des Gewichtes gewandert seyn. [...] Was wir in der Gegenwart von jener Gattung Musik besitzen, stammt meistens aus den Bibliotheken der berühmten Fugger [...]. Dies beweist, dass die Tonkunst, in den Augen der Profanen von jeher unter ihren Mitschwestern auf die letzte Rangstufe verwiesen, noch immer der verdienten Erhöhung und Würdigung entgegenharrt. ${ }^{\text {“17 }}$

\section{Die kaiserlichen Privatsammlungen - am Beispiel der „Leopoldina“}

Um einen Eindruck von den kaiserlichen Musiksammlungen zu geben, die unter Graf Dietrichstein im Zeitraum nach 1826 der Hofbibliothek eingegliedert wurden, sei als pars pro toto ein prominenter Teilbestand herausgegriffen, die Privatsammlung Kaiser Leopolds I. (1640-1705), die als „Schlafkammerbibliothek“ (bibliotheca cubicularis) bekannt wurde. Bereits das äußere Erscheinungsbild der Bände lässt erkennen, dass Leopold ihnen besondere Bedeutung beimaß: Er ließ sie in weißes Pergament mit Goldprägung binden; auf der Vorderseite findet sich meist das charakteristische Porträt des Kaisers als Supralibros, auf der Rückseite der Doppeladler, gelegentlich mit Leopolds Wahlspruch „Consilio et industria“. Insgesamt 524 Bände umfasst diese Sammlung; sie enthält Werke von 58 verschiedenen Komponisten, 41 anonyme Kompositionen und 15 Sammelhandschriften. ${ }^{18}$ Der Name "Schlafkammerbibliothek“ deutet ebenfalls auf die hohe persönliche Wertschätzung dieser Sammlung durch Leopold hin: Das „Cubicularium“, das zu den intimsten Aufenthaltsorten des Herrschers zählte, befand sich im ersten Stock des „Schweizertraktes“ der Wiener Hofburg, hatte eine Fläche von circa 150 Quadratmetern und diente nicht nur der Unterbringung der kaiserlichen Privatbibliothek, sondern auch der Veranstaltung von Musik- und Theateraufführungen.

Das Repertoire dieser Sammlung umfasst einerseits Kirchenmusik (darunter auch Oratorien und Sepolcri), andererseits die bei Hof aufgeführten musikdramatischen Werke; es bildet weiters die von Leopold getroffene Auswahl der zeitgenössischen Komponisten ab. Leopold übernahm zunächst die Kapelle seines Vaters, verzichtete auf größere Umbesetzungen und baute sie allmählich zu dem hochentwickelten Klangkörper auf, der das Rückgrat der legendären musikalischen Festereignisse bei Hof bilden sollte. Der erste Kapellmeister war Antonio Bertali, der bereits zu Zeiten Ferdinands III. die Leitung innegehabt hatte. Ihm folgte 1669 Felice Sances und 1679 Johann Heinrich Schmelzer, der aber bereits 1680 der großen Pestepidemie zum Opfer fiel. Erst nach zweijähriger Vakanz wurde Antonio Draghi zum Hofkapellmeister ernannt (er hatte dieses Amt aber bereits seit Schmelzers Tod interimistisch ausgeübt); er leitete die Hofmusikkapelle bis zu seinem Tod 1700. Als sein Nachfolger wurde Antonio Pancotti bestellt, der seit 1665 als Sopra-

17 Ebd., S. 102.

18 Josef Gmeiner, „Die ,Schlafkammerbibliothek' Kaiser Leopolds I.“, in: Biblos. Beiträge zu Buch, Bibliothek und Schrift 43 (1994), S. 199-211. 
nist an der Hofmusik mitgewirkt hatte und seit 1697 das Amt des Vizekapellmeisters bekleidete. Man sieht an dieser Abfolge der leitenden Personen, dass italienische Musiker den Ton angaben. Viele Angehörige der Hofmusikkapelle waren auch Komponisten, und auch hier bildeten die Italiener (17) im Vergleich mit den Österreichern und Deutschen (neun) die klare Mehrheit.

\section{Von der inhaltlichen zur institutionellen Identität der Musiksammlung}

Die Musikaliensammlung, die in den Jahren nach 1826 innerhalb des Gesamtbestandes der Hofbibliothek entstand, war zunächst nichts anderes als eine inhaltliche Bestandskonzentration ohne jede institutionelle oder lokale Eigenständigkeit. Sie verdankt ihre Entstehung dem persönlichen Interesse und der Zuwendung durch Moritz Graf Dietrichstein und der Besetzung wichtiger Bibliotheksstellen durch musikkundige Gelehrte wie Ignaz von Mosel und Anton Schmid. Nach dem Ende der Ära Dietrichstein (1845) ebbte das Interesse an Musik innerhalb der Bibliothek merkbar $\mathrm{ab}$, und als im Verlauf der folgenden Jahre die Erwerbung bedeutender Autographenbestände von Mozart und Beethoven möglich wurde, übte sich die Hofbibliothek in Zurückhaltung - was zu dem Umstand führte, dass die Königliche Bibliothek zu Berlin (die heutige Staatsbibliothek) an Mozart- und Beethovenerwerbungen ihre Wiener Schwesterinstitution weit in den Schatten stellte. Dass Musik weiterhin als unselbstständiges Bestandssegment gesehen wurde, zeigte sich nachdrücklich bei der umfassenden Neukatalogisierung sämtlicher Handschriften des Hauses gegen Ende des 19. Jahrhunderts: Die Musikhandschriften wurden den allgemeinen Handschriften beigeordnet und umfassten den Signaturenbereich Cod. 15501 bis 19500.

Erst 1906 erhielt die Musiksammlung einen eigenen Lesesaal und drei Depot-Räume, allerdings immer noch im Verband des historischen Bibliotheksbereiches auf dem Wiener Josefsplatz. Einen wichtigen Schritt zur Verselbstständigung machte sie erst 1920 mit dem Umzug in den vierten Stock des Albertinagebäudes, wo sie im Verlauf der folgenden Jahrzehnte eine bedeutende inhaltliche und räumliche Ausweitung erlebte. 2005 erfolgte die bislang letzte Übersiedlung in das Palais Mollard in der Wiener Herrengasse, wo nunmehr auch die Sicherheitsbedingungen und konservatorischen Standards dem Rang einer der großen europäischen Musiksammlungen entsprechen. 